\author{
Italique
}

XV | 2012

Varia

\title{
Echi petrarcheschi in «Superbi colli, e voi sacre ruine» di Baldassarre Castiglione
}

\section{Hans Honnacker}

\section{(2) OpenEdition}

\section{Journals}

\section{Edizione digitale}

URL: http://journals.openedition.org/italique/352

DOI: $10.4000 /$ italique.352

ISSN: 1663-4438

\section{Editore}

Librairie Droz

\section{Edizione cartacea}

Data di pubblicazione: 1 dicembre 2012

Paginazione: 163-179

ISBN: 978-2-600-01639-1

ISSN: 1423-3983

\section{Notizia bibliografica digitale}

Hans Honnacker, « Echi petrarcheschi in «Superbi colli, e voi sacre ruine» di Baldassarre Castiglione », Italique [Online], XV | 2012, online dal 01 décembre 2015, consultato il 19 avril 2019. URL : http:// journals.openedition.org/italique/352 ; DOI : 10.4000/italique.352 
HANS HONNACKER

\author{
ECHI PETRARCHESCHI IN \\ «S U P ERB I C OLLI, \\ E VOI SACRE RUINE» \\ D I B A L D A S ARRE CASTIGLIONE
}

A Marco Romanelli, amico e compagno di tanti viaggi letterari 



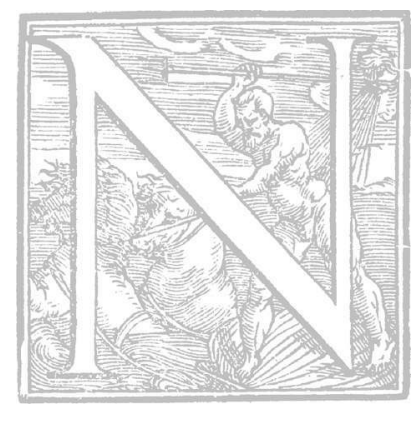

el dialogo Non sperate di liberarvi dei libri, Umberto Eco e Jean-Claude Carrière discutono sul 'filtraggio' dei libri, delle opere letterarie nei secoli, cioè su come alcuni autori e/o testi letterari vengano giustamente o ingiustamente dimenticati nel corso del tempo:

JCC [Jean-Claude Carrière] Ti citavo i nostri grandi poeti francesi sconosciuti. Parlami degli autori italiani dimenticati. Ingiustamente dimenticati.

UE [Umberto Eco] Ho parlato dei poeti barocchi minori, anche se il più importante di loro probabilmente, Giovan Battista Marino, all'epoca fu più famoso in Francia che in Italia. Per il resto del XVII secolo, i nostri grandi uomini erano scienziati e filosofi, come Galileo, o Bruno, o Campanella, che appartenevano al "sillabo" universale. Per quanto il nostro XVIII secolo sia stato molto debole, paragonato a ciò che succedeva in Francia nello stesso periodo, non possiamo tuttavia trascurare il caso di Goldoni [...].

JCC [Jean-Claude Carrière] [...] Ma voglio aggiungere qualcosa a quel che dicevi riguardo al XVIII secolo [...]. I versificatori hanno, ovviamente, prodotto e pubblicato migliaia di versi, milioni forse, ma nessun francese è capace di citare una sola di queste poesie. Ti posso citare Florian, che è un affabulatore mediocre, l'abate Delille, Jean-Baptiste Rousseau, ma chi li ha letti e chi, soprattutto, potrebbe leggerli oggi? [...] Perché questi "poeti", o coloro che si credevano tali, si accontentavano di applicare le regole del secolo precedente, fissate da Boileau. Mai si sono scritti tanti versi e mai così poche poesie. Non una sola, in più di un secolo. Quando ti accontenti di applicare le regole, ogni sorpresa, ogni scintillio, ogni ispirazione evapora [...].

UE [Umberto Eco] In questo preciso caso, il filtraggio è positivo. È meglio non ricordarsi di questi "poeti" di cui hai parlato.

JCC [Jean-Claude Carrière] Sì, è stato un filtraggio implacabile e giusto, in questo caso. Tutti nel vortice dell'oblio [...]. ${ }^{\mathrm{I}}$

\section{L'oblio della poesia latina ed italiana di Castiglione}

Viene da chiedersi: è questo il motivo per cui le poesie latine ed italiane di Baldassarre Castiglione sono state dimenticate? Perché si è acconten- 
tato di applicare le regole stabilite da Francesco Petrarca, e canonizzate da Pietro Bembo nelle Prose della volgar lingua (I525)? E ci ricorderemmo del conte come autore, se non avesse scritto Il Libro del Cortegiano (I528) che l'ha reso famoso in tutta Europa fino ad oggi? Le sue poesie sono quindi sopravissute grazie all'ombra 'protettiva' del suo capolavoro? Ed infine Castiglione pensava di diventare famoso per la sua produzione lirica (come credeva ad esempio il giovane Erasmo), ${ }^{2}$ per il suo dialogo sul 'bon cortigiano' o per le sue epistole? Nel dialogo succitato Eco sostiene a proposito di Petrarca:

UE [Umberto Eco] È la stessa storia di Petrarca. Ha passato la vita lavorando alla sua grande opera in latino, Africa, convinto che sarebbe diventata la nuova Eneide e che gli avrebbe portato gloria. Quando non aveva niente di meglio da fare scriveva invece i sonetti, che lo hanno reso famoso per sempre. ${ }^{3}$

Queste e altre potrebbero essere dunque le domande-guida della presente indagine sulla 'sfortuna' letteraria della produzione lirica di Castiglione in generale, e della poesia Superbi colli, e voi sacre ruine in particolare.

\section{La produzione lirica latina ed italiana di Castiglione}

La produzione lirica latina di Castiglione che conta una ventina di poesie, ${ }^{4}$ assai apprezzate all'epoca e dai critici classicisti, ${ }^{5}$ rappresentava sicuramente molto di più del solito 'tirocinio' di ogni umanista che si rispettasse. Di diverso avviso è Bruno Maier nella sua introduzione a Il Libro del Cortegiano con una scelta delle Opere minori di Baldesar Castiglione, anche se ammette qualche originalità nei Carmina del conte:

Secondo noi, è ben vero che in diversi componimenti latini del Castiglione non c'è molto più dell'esercizio di stile; ma è altrettanto vero che in alcuni di essi ci sono una sincera ispirazione ed un sentimento animatore, che si traducono in qualcosa di vivo, di commosso e di delicato. ${ }^{6}$

Maier cita qui a titolo di esempio l'elegia De Elisabella Gonzaga canente, «che, pur attraverso il travestimento virgiliano, attesta la 
devota ammirazione del Castiglione per la bella signora di Urbino e ci riporta, per taluni aspetti, al mondo del Cortegiano [...]». ${ }^{7}$

La produzione lirica italiana, molto acclamata anch'essa all'epoca, consisteva complessivamente solo in dieci sonetti e quattro canzoni. ${ }^{8}$ Scrive Vittorio Cian al riguardo:

Figlio del suo tempo, [Castiglione] doveva considerare l'esercizio della poesia quasi un ornamento doveroso per un gentiluomo della sua qualità, vivente in una corte dove la poesia contava non pochi amatori e cultori. Dilettante serio e modesto e buongustaio, fu spinto verso il Parnaso, per fortuna sua, non da un proposito prettamente letterario o dagli allettamenti della nuova moda del petrarchismo risorgente, ma soprattutto da un impulso interiore, schiettamente sentimentale, l'ammirazione e la devozione amorosa per quella duchessa Elisabetta, che sappiamo già essere la sua Musa ispiratrice. ${ }^{9}$

\section{Il sonetto Superbi colli, e voi sacre ruine}

Ma vediamo più da vicino il sonetto Superbi colli, e voi sacre ruine, composto probabilmente nella Roma di Leone $X\left(\right.$ III3-2I): ${ }^{\text {IO }}$

Superbi colli, e voi sacre ruine, che 'l nome sol di Roma ancor tenete, ahi, che reliquie miserande avete di tant'anime eccelse e pellegrine!

Colossi, archi, teatri, opre divine, trionfal pompe gloriose e liete, in poco cener pur converse siete e fatte al vulgo vil favola al fine.

Così, se ben un tempo al tempo guerra fanno l'opre famose, a passo lento e l'opre e i nomi il tempo invido atterra.

Vivrò dunque fra' miei martir contento: ché se 'l tempo dà fine a ciò ch'è in terra, darà forse ancor fine al mio tormento. ${ }^{11}$

\section{Maier commenta cosi questa poesia di Castiglione:}

Un interesse particolare presenta il sonetto Superbi colli, e voi sacre ruine, che sembra ispirato dall'umanistica commozione per la grandezza passata di 
Roma e dal senso fatale e inesorabile trascorrere del tempo. In verità, questi due motivi, che in qualche animo romanticamente disposto potrebbero destare una suggestione profonda, e far pensare magari a quella "poesia delle rovine», che tanta fortuna avrà dal preromanticismo al Carducci, sono espressi non senza una certa enfasi retorica; e costituiscono, per di più, una lunga similitudine, cui l'autore ricorre per alludere al proprio «tormento» (che resta pur sempre un generico e indefinito «tormento»), nella speranza che il tempo, il quale "dà fine a ciò ch'è in terra», tale tormento, prima o poi, faccia cessare. Si tratta, dunque, d'un sonetto di tono triste e dolente, ma svolto più con l'ingegnosa abilità del letterato (si pensi al prebarocco inciso «se ben un tempo al tempo guerra Fanno l'opre famose»), che con la lirica spontaneità del poeta. Sicché a ragione il Croce ne diede un severo giudizio. ${ }^{{ }^{2}}$

Sulla scia di Benedetto Croce, ${ }^{\mathrm{I}}$ Maier accusa quindi questo componimento di scarsa «lirica spontaneità del poeta». Dietro tale giudizio sta evidentemente la concezione crociana della poesia come ispirazione ed originalità che, tuttavia, ha poco a che fare con la poetologia del Cinquecento che, come è ben noto, vedeva nel principio oraziano dell'imitatio auctorum ${ }^{\mathrm{I}}{ }^{4}$ la via maestra della poesia. ${ }^{\mathrm{IS}}$ Pertanto la poesia di Castiglione va giudicata secondo i parametri della poetica dell'epoca, $e$ non secondo un concetto nemmeno tanto velatamente romantico di poesia che pretende la «sincera ispirazione ed un sentimento animatore, che si traducono in qualcosa di vivo». Nell'ottica dell'imitatio auctorum (e dell'aemulatio), è proprio «l'ingegnosa abilità» di Castiglione, di cui parla Maier, pur con una accezione negativa, che rende particolare questo sonetto.

Il motivo «del fatale e inesorabile trascorrere del tempo» non è ovviamente nuovo, e risale al verso virgiliano Sed fugit interea, fugit inreparabile tempus (Virgilio, Georgiche, III 284) o, per quanto riguarda le rovine di Roma, al Sic transit gloria mundi (Agostino Patrizi Piccolomini, Caerimoniale Romanum ([I488]/I5I6), I, 2, 3 e Matteo, 24, 35). Tanti poeti, da Petrarca a Jacopo Sannazaro, banno ripreso il motivo delle rovine dell'antica Roma, come indica lo stesso Maier nelle sue note al testo. Ricordiamo qui anche la parodia che ne fa Ludovico Ariosto nel suo Orlando Furioso, nel famoso episodio del viaggio sulla luna intrapreso da Astolfo ("Vide un monte di tumide vesiche, / che dentro parea aver tumulti e grida; / e seppe ch'eran le corone antiche / e degli Assirii e de la terra lida / e de' Persi e de' 
Greci, che già furo / incliti, et or n'è quasi il nome oscuro // [...] // Ruine di cittadi e di castella / stavan con gran tesor quivi soz?:ppra»). ${ }^{16}$

Nondimeno è proprio Petrarca l'autore che Castiglione intende di imitare (e superare) qui, ovvero il Petrarca del Canzoniere ma anche quello dei Trionfi. Vediamo più nel dettaglio $i$ rapporti intertestuali tra questo sonetto del Castiglione e alcune poesie petrarchesche.

Per il motivo delle rovine dell'antica Roma, Maier indica nelle note come testo di riferimento fra gli altri, ${ }^{17}$ la nota canzone petrarchesca Spirto gentil, che quelle membra reggi (Rvf 53, dedicata forse a Cola di Rienzo o, come suggerisce Marco Santagata nel suo commento, a Bosone da Gubbio), in particolare i versi 29-36:

L'antiche mura ch'anchor teme et ama et trema 'l mondo, quando si rimembra del tempo andato e 'ndietro si rivolve, e i sassi dove fur chiuse le membra di ta' che non saranno senza fama, se l'universo pria non si dissolve, et tutto quel ch'una riuna involve, per te spera saldar ogni suo vitio. ${ }^{18}$

Tuttavia è innanzitutto il Trionfo del Tempo di Petrarca da cui $i$ versi 5-7 del sonetto castiglionesco ("Colossi, archi, teatri, opre divine, / trionfal pompe gloriose e liete, / in poco cener pur converse siete») prendono spunto:

Passan vostri trionfi e vostre pompe,

Passano le signorie, passano i regni,

Ogni cosa mortal Tempo interrompe. ${ }^{19}$

Proprio su questo motivo del tempo che interrompe e fa dimenticare tutto, Castiglione incentra la prima terzina con «l'ingegnosa abilità, come direbbe Maier:

Così, se ben un tempo al tempo guerra fanno l'opre famose, a passo lento e l'opre e i nomi il tempo invido atterra.

Pertanto, nell'ottica dell'imitatio (e dell'aemulatio), il nostro poeta riprende e supera con virtuosismo il modello petrarchesco. Basti pensare 
solo alla triplice ripetizione del lemma tempo in tre versi consecutivi, collegati da enjambement, che rende efficace il concetto della fugacità delle cose terrene.

\section{Il petrarchismo ne Il Libro del Cortegiano ${ }^{\mathrm{I} 9}$}

Il modello petrarchesco viene suggerito al Castiglione, come scrive Maier, "dall'autorità teorica, oltre che dall'esempio diretto, del Bembo, dittatore del petrarchismo letterario e amico carissimo del Castiglione»》. ${ }^{2}$ Non stupisce che nella versione definitiva del Cortegiano del I528, Pietro Bembo sia uno dei locutori dominanti che, nel quarto libro, elabora la sua concezione neoplatonica dell'amore, in cui Castiglione riprende in larga parte l'argomentazione degli Asolani (I505) dello stesso Bembo. ${ }^{22}$

\section{I La questione della lingua nel Cortegiano}

Nondimeno Bembo (e con lui Petrarca) è auctoritas non solo per quanto concerne il tema dell'amore cosi come viene sviluppato nel Cortegiano, ma anche per quanto riguarda la lingua del perfetto cortigiano. Come è noto, nel primo libro del Cortegiano, viene discussa la cosiddetta questione della lingua, in cui Federico Fregoso difende la posizione bembiana del toscano trecentesco di Petrarca e Boccaccio. ${ }^{23}$ Ludovico da Canossa, il locutore 'dominante' del primo libro, invece assume la posizione di un volgare italiano moderno e sovrarregionale (posizione attribuita al Castellano, I529, di Gian Giorgio Trissino), mentre Giuliano de' Medici, come già nelle Prose della volgar lingua (I525), si esprime a favore di un toscano moderno (posizione attribuita al Discorso o dialogo intorno alla nostra lingua, Is24, di Niccolò Machiavelli ed a Il Cesano, del la lingua Toscana, I524-I525, di Claudio Tolomei):

«Io già l'ho detto,» rispose il Conte; «ma, parlandosi della lingua toscana, forse più saria debito del signor Magnifico che d'alcun altro il darne la sentenzia». Disse il Magnifico: «Io non posso né debbo ragionevolmente contradir a chi dice che la lingua toscana sia piú bella dell'altre. È ben vero che molte parole si ritrovano nel Petrarca e nel Boccaccio, che or son interlassate dalla consuetudine d'oggidì; e queste io, per me, non 
usarei mai, né parlando né scrivendo; e credo che essi ancor, se insin a qui vivuti fossero, non le usarebbono più̀. Disse allor messer Federico: «Anzi le usarebbono; e voi altri, signori Toscani, dovreste rinovar la vostra lingua, e non lassarla perire, come fate; ché ormai si po dire che minor notizia se n'abbia in Fiorenza, che in molti altri lochi della Italia». Rispose allor messer Bernardo: «Queste parole che non s'usano più in Fiorenza, sono restate ne' contadini e, come corrotte e guaste dalla vecchiezza, sono dai nobili rifiutate [...]». Allora la signora Duchessa, «Non usciam,» disse, «dal primo proposito e facciam che 'l conte Ludovico insegni al cortegiano il parlare e scriver bene, e sia o toscano o come si voglia». ${ }^{24}$

Benché sembri prevalere la posizione di Ludovico da Canossa, la discussione sulla questione della lingua viene sospesa da Emilia Pio, 'Iuogotenente' della duchessa di Urbino, e quindi rimandata:

Allor la signora Emilia, «A me par,» disse, «che questa vostra disputa sia mo troppo lunga e fastidiosa; però fia bene a differirla ad un altro tempo». Messer Federico pur incominciava a rispondere; ma sempre la signora Emilia lo interrompeva. ${ }^{25}$

La posizione di Castiglione a proposito della questione della lingua non è quindi cosi chiara, come viene invece generalmente affermata nella critica. ${ }^{26}$ Nella seconda edizione del Cortegiano (I520-I52I), Emilia Pio dava addirittura l'incarico a Bembo di continuare la discussione, rimandandola ad un'altra serata:

Rispose ridendo la signora madonna Emilia: «Pena la disgrazia mia a qual di voi per ora parla più di questa materia, perché voglio che la rimettiamo ad un'altra sera, et il parlarne sia in tutto impresa di messer Piero Bembo». Disse el conte: «Io son contentissimo, anzi di tutto a lui mi rimetto e lo faccio iudice». Rispose messer Federico: «Et io ancora». Rise messer Piero e disse: «E s'io non volessi accettare questo carico?». Rispose madonna Emilia: «Ve lo commanderò per l'auttorità concessami dalla signora Duchessa e non potrete negarlo». ${ }^{26}$

Castiglione accenna qui evidentemente alle Prose della volgar lingua di Bembo, che sarebbero uscite da li a poco, nel I525. Pertanto anche nel caso della questione della lingua, la posizione bembiana rimane quella di riferimento, dalla quale nessuno può prescindere. Non a caso lo stesso 
Castiglione, nella versione definitiva del Cortegiano del I528, si adegua ai dettami poetologici e linguistici di Bembo e quindi al suo petrarchismo.

\subsection{Le poesie nel Cortegiano}

D'altronde, Petrarca non è soltanto il modello poetologico difeso da Federico Fregoso nella sopra citata questione della lingua, ma viene chiamato in causa anche in altri luoghi della discussione sulla formazione del bon cortigiano. Nel suo dibattito con Ludovico Canossa sulla questione della superiorità delle armi sulle lettere nella formazione, Bembo cita i seguenti versi petrarcheschi: ${ }^{28}$

Giunto Alessandro alla famosa tomba del fero Achille, sospirando disse:

$\mathrm{O}$ fortunato, che sì chiara tromba trovasti e chi di te sì alto scrisse! ${ }^{29}$

Bembo riporta qui questi famosi versi di Petrarca per dare autorevolezza alla sua posizione della superiorità delle lettere sulle armi. Alla fine Ludovico Canossa è costretto a venire incontro alla posizione bembiana, affiancando le lettere alle armi nella formazione del cortigiano:

Rispose allora il Conte: «Io biasmo i Franzesi che estiman le lettre nuocere alla profession dell'arme, e tengo che a niun più si convenga l'esser litterato che ad un om di guerra; e queste due condizioni concatenate, e l'una dall'altra aiutate, il che è convenientissimo, voglio che siano nel nostro cortegiano: né per questo parmi esser mutato d'opinione. Ma, come ho detto, disputar non voglio qual d'esse sia piú degna di laude».3०

La lezione di Petrarca si vede infine anche in un altro sonetto che l'Unico Aretino (Bernardo Accolti) ${ }^{3 \mathrm{I}}$ recita in onore della duchessa Elisabetta Gonzaga di cui, tuttavia, Castiglione non riporta il testo, dando per scontato che $i$ suoi lettori lo conoscano:

Consenti, o mar di bellezza e virtute, ch'io servo tuo, sia d'un gran dubbio sciolto, se l'S che porti nel candido volto, 
significa mio stento o mia salute,

se dimostra soccorso, o servitute, sospetto, o securtà, secreto, o stolto, se speme, o strido, se salvo, o sepolto! Se le catene mie strette, o solute; ch'io temo forte che non mostri segno de superbia, sospir, severitate, strazio, sangue, sudor, supplicio e sdegno.

Ma se loco ha la pura veritate, questa $\mathrm{S}$ dimostra con non poco ingegno un sol solo in bellezza e 'n crudeltate. ${ }^{32}$

Questo sonetto assai applaudito dai personaggi de Il Libro del Cortegiano $^{33}$ (anche se non improvvisato come l'Unico Aretino vorrebbe far credere) è il tipo di imitazione poetica ingegnosa a cui si ispira anche Castiglione nel suo sonetto Superbi colli, e voi sacre ruine, seguendo e superando, come si è visto, il modello petrarchesco. ${ }^{34}$

Prima di recitare la sua poesia, l'Unico Aretino aveva proposto come gioco di discussione la decifrazione del monile a forma di $\mathrm{S}$ che la duchessa era solita portare in fronte e che si vede nel celebre ritratto della duchessa, attribuito a Raffaello. ${ }^{35}$

\section{Conclusioni provvisorie}

In questa breve panoramica incentrata sulla 'sfortuna' letteraria della produrione lirica di Castiglione in generale, e della poesia Superbi colli, e voi sacre ruine in particolare, si è sostenuta la tesi che questo sonetto debba essere gindicato secondo $i$ parametri della poetica dell'epoca, e non secondo un concetto crociano di poesia che pretende "sincera ispirazione ed un sentimento animatore, che si traducono in qualcosa di vivon, come scriveva Maier. Nell'ottica dell'imitatio auctorum (e dell'aemulatio), è proprio «l'ingegnosa abilità» di Castiglione di imitare il modello petrarchesco, che rende particolare il suo componimento anche all'interno della sua produzione lirica. Quindi, a mio avviso, il sonetto Superbi colli, e voi sacre ruine, forse il componimento lirico più celebre di Castiglione almeno a giudicare dalla sua 'fortuna' al livello europeo, dovrebbe essere rivalutato in tale ottica (cosi come d'altronde tutta la produ₹ione lirica di Castiglione sia latina 


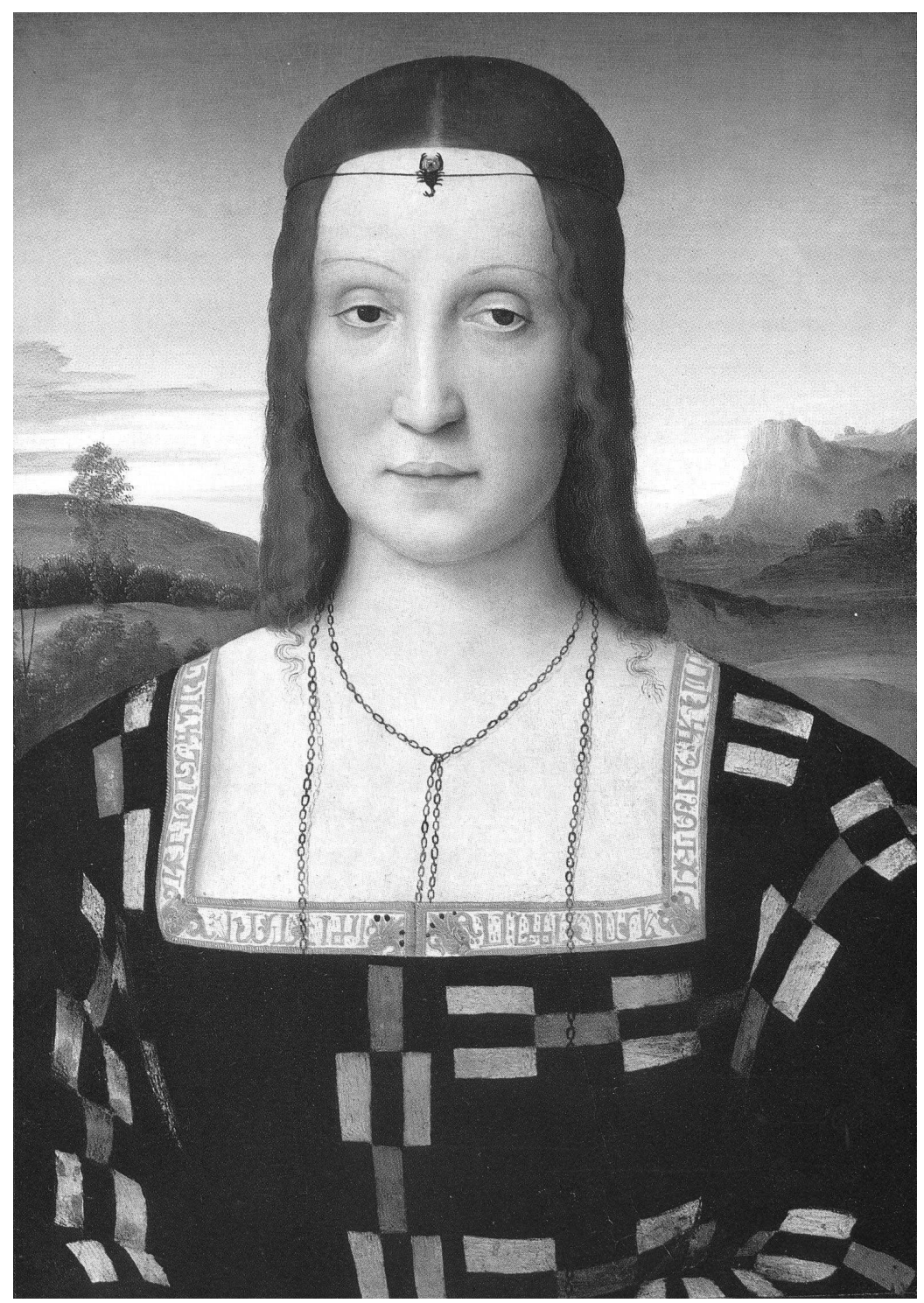

Raffaello (attribuito)

Elisabetta Gonzaga, i 505 ca.

che italiana), e fatto uscire dall'ingombrante' ombra del Cortegiano, e non soltanto in quanto capostipite della cosiddetta 'poesia delle rovine. ${ }^{36}$

Hans Honnacker 


\section{Riferimenti bibliografici}

\section{Bastiaensen 2003}

Bastiaensen, M., Jean Flemingue et Baldesar Castiglione: une voix néolatine dans un chcur plurilingue, in Hommages à Carl Deroux, vol. s: Christianisme et Moyen Âge. Néolatin et survivance de la latinité, Bruxelles, Defosse, 2003, pp. 2869I.

Berni/Castiglione/Della Casa i 995

Berni, F. / Castiglione, B. / Della Casa, G., Carmina, a cura di M. Scorsone, Torino, Edizioni Res, I995.

Carrière/Eco 2009

Carrière, J.-C. e Eco, U., Non sperate di liberarvi dei libri, Milano, Bompiani, 2009 (trad. dal francese).

Castiglione i 968

La seconda redazione del "Cortegiano" di Baldassarre Castiglione, a cura di G. Ghinassi, Firenze, I968.

Castiglione $198 \mathrm{I}^{3}$

Il Libro del Cortegiano con una scelta delle Opere minori di Baldesar Castiglione, a cura di B. Maier, Torino, UTET, $198 \mathrm{I}^{3}$.

Cian I95 I

Cian, V., Un illustre nunzio pontificio del Rinascimento: Baldassar Castiglione, Città del Vaticano, I95 I.

\section{Croce i 991}

Croce, B., Poesia popolare e poesia d'arte. Studi sulla poesia italiana dal Tre al Cinquecento, Ercolano, Bibliopolis, I99I.

Hempfer 2004

Hempfer, K.W., Letture discrepanti. La ricezione dell'Orlando Furioso nel Cinquecento. Lo studio della ricezione storica come euristica dell'interpretazione, trad. di H. Honnacker, Modena, Panini, 2004.

Honnacker $2002 \mathrm{a}$

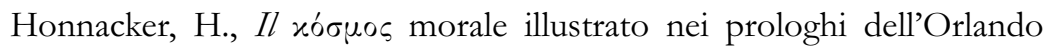
Furioso di Ludovico Ariosto nelle edizioni del IsI6 e del I52I: la Weltanschanung ariostesca fra Orazio ed Erasmo, in «Schifanoia», XXII (2002), pp. 3 I-54. 
Honnacker $2002 \mathrm{~b}$

Honnacker, H., Der literarische Dialog des primo Cinquecento. Inszenierungsstrategien und 'Spielraum', Baden-Baden, Koerner, 2002.

Honnacker 2003

Honnacker, H., Balthasar Castilioneus, in C.A.L.M.A.: Compendium Auctorum Medii 'vii (500-I500), a cura di di Michael Lapidge, Gian Carlo Garfagnini e Claudio Leonardi, I.s, Firenze, SISMEL-Edizioni del Galluzzo, 2003 .

Honnacker 20I I

Honnacker, H., Erasmus Roterodamus (Quaderni di C.A.L.M.A. I), Firenze, SISMEL-Edizioni del Galluzzo, 20 I I.

Morel-Fatio I 894

Morel-Fatio, A., Histoire d'un sonnet, in «Revue d'Histoire Littéraire de la France», I (1 894), pp. 97-102.

Morel-Fatio I 903

Morel-Fatio, A., A propos du sonnet 'Superbi colli', in «Bulletin Italien», III (1903), pp. 37-38.

Morel-Fatio I 904

Morel-Fatio, A., Histoire de deux sonnets, in A. Morel-Fatio, Etudes sur l'Espagne, $3^{\mathrm{e}}$ série, Paris, Bouillon, I 904, pp. I4I-64.

Mutini 1979

Mutini, C., Baldassarre Castiglione, in DBI, vol. XXII (I 979), pp. 53-68.

Petrarca i 996

Petrarca, F., Il Canzoniere, a cura di M. Santagata, Milano, A. Mondadori, I 996 .

Profeti 2003

M.G. Profeti, M.G., "Yo vi la grande y alta jerarquia": el tema de las ruinas en Quevedo, in Esteba el jardin en flor: Homenaje a Stefano Arata, in "Criticón» 87-89 (2003), pp. 709-I 8.

Profeti 2009

Profeti, M.G., Commedie, riscritture, libretti: la Spagna e l'Europa, Firenze, Alinea, 2009.

Roncaccia 2006

Roncaccia, A., Il metodo critico di Ludovico Castelvetro, Roma, Bulzoni, 2006. 


\author{
Weinberg (1 974²) \\ Weinberg, B., History of Literary Criticism in the Italian Renaissance, 2 voll., \\ Chicago, $1974^{2}$.
}

\title{
Note
}

I. Carrière/Eco 2009, pp. 8 I-84.

2. Per Erasmo si veda Honnacker 20 I I, p. XII e la bibliografia ivi riportata.

3. Carrière/Eco 2009, p. 96.

4. Per la produzione lirica latina di Castiglione si veda Honnacker 2003, pp. $587-90$ e la bibliografia ivi riportata.

5. Nella sua Ragion poetica (I, XLII), Gian Vincenzo Gravina (I664-I7 I8) le paragonò addirittura alle poesie di Virgilio, Catullo e Tibullo.

6. Castiglione i $98 \mathrm{I}^{3}$, p. 44.

7. Ibidem.

8. Cfr. Cian i95 I, p. I99.

9. Cian I95 I, p. 198.

Io. Cian i95 I, p. 204.

i i. Castiglione i98 ${ }^{3}$, pp. 599-600. Cian riporta l'autografo del sonetto in una versione precedente: «Superbi colli et voi sacre ruine / Ch'el nome sol di roma ancor tenete / Ah che relliquie miserande havete / Di tante anime excelse et pellegrine, // Theatri, Archi, Colossi, opre divine, / Triomphal pompe gloriose e liete, / In poche cener pur confuse sete, / Et fatt'al volgo vil favol'al fine. // Così seben un tempo al tempo guerra / Fanno l'opre famose a passo lento / Et l'opre e i nomi insieme il tempo atterra. // Vivrò dunque fra miei martir contento, / Che s'el tempo dà fine ad ciò ch'è in terra, / Darà forse ancor fine al mio tormento» Cian I95 I, p. 206, nota 2.

i 2. Castiglione i98 $\mathrm{I}^{3}$, p. 43. A dire il vero, anche Cian dà un giudizio poco lusinghiero: «Invece, in un altro sonetto, troppo più famoso di questi due, il Castiglione, più tardi, nella Roma di Leone $\mathrm{X}$, volle riprendere e svolgere con un'ampiezza pericolosa questo sentimento, degradato a concetto, quello del tempo distruttore delle cose terrene, applicandolo alle rovine dell'Urbe e al suo proprio caso di poeta tormentato dall'Amore. È il sonetto Superbi colli e voi, sacre ruine, composto probabilmente sotto l'influsso di esempî contemporanei, quali quelli del Sannazaro, di Serafino dell'Aquila e dell'Unico Accolti che si aggiungevano al Petrarca [....]. Gli nuoce, anzittutto, il tono enfatico che il poeta assume sin dal principio, quasi montando sui trampoli, per rivolgere una sonora allocuzione ai colli e alle rovine di Roma. Ma non meno gli nuoce la sproporzione 
conseguente fra i due termini contrapposti, quello delle rovine romane e della fine inesorabile d'ogni cosa quaggiù per l'opera distruttrice del tempo, e quello del suo tormento amoroso. Infatti, mentre il primo si svolge invadente per gran parte del componimento e con una battuta di pretto conio presecentesco, degna di Serafino Aquilano e dell’Unico (“[...] se ben un tempo al tempo guerra - Fanno l'opre famose [...]"), il secondo rimane strozzato in un finale che più freddamente concettoso non potrebbe essere: "Vivrò dunque fra' miei martir contento: / ché se 'l tempo dà fine a ciò ch'è in terra, / darà forse ancor fine al mio tormento." Con quel "forse" dell'ultimo verso il poeta ammetteva, a quanto pare, la possibilità minacciosa che il suo tormento si protraesse perfino nell'oltretomba; un tormento, dunque, senza fine!» Cian I95 I, pp. 204-205. In contrasto con il giudizio negativo espresso da Cian, Maier e da quasi tutta la critica letteraria novecentesca in Italia (si veda, a titolo di esempio, il giudizio piuttosto 'freddo' di Mutini 1979, in particolare p. 66), questo sonetto ha riscosso una discreta celebrità europea, in quanto capostipite della cosiddetta 'poesia delle rovine' ed imitato da autori illustri quali Joachim du Bellay, Félix Lope de Vega, Paul Scarron ed altri (a tale proposito si vedano Morel-Fatio i 894, Morel-Fatio I903, Morel-Fatio i 904 e Bastiaensen 2003). Per la 'fortuna' del sonetto castiglionesco in particolare in Spagna si confrontino Profeti 2003 e Profeti 2009, p. I 8, nota Io.

I 3. «Il Castiglione non era il Bembo e aveva altra pienezza di forma e scioltezza di movimenti. Pure, il suo sonetto, tante volte imitato, sulle rovine di Roma, non ha una sola pennellata veramente calda e sentita, e la chiusa nella quale (come scriveva, ammirando, il Giovio) «amatorii doloris finem superba comparatione desperat», è insieme artificiosa e meschina [...]» Croce i991, pp. 375-76.

I4. «Aut famam sequere aut sibi convenientia finge / scriptor [...] / [...] vos exemplaria Graeca / nocturna versate manu, versate diurna» Orazio, Ars poetica, vv. II9 sg. e 268 sg.

I 5. La poetica dell'imitatio auctorum come viene concepita da Orazio nella sua cosiddetta Ars poetica è da considerare norma poetica vincolante per tutto il Quattrocento ed oltre, anche allorché, solo a cominciare dalla seconda metà del Cinquecento, la riscoperta della Poetica aristotelica sembra prevalere sulla poetica della tradizione retorico-oraziana. A tale proposito si vedano Weinberg $1974^{2}$, vol. I, pp. 7 I sgg., I I I-55 e 349 sgg. e Hempfer 2004, pp. 50 sgg.

i6. Ariosto, Orlando Furioso, XXXIV, LXXVI, 3 - LXXIX, 2 (al riguardo si confronti Honnacker 2002a, in particolare p. 44).

17. Maier cita anche il carme latino dello stesso Castiglione De morte Raphaelis pictoris: «Quod lacerum corpus medica sanaverit arte, / Hippolytum Stygiis et revocaverit aquis, // Ad Stygias ipse est raptus Epidaurius undas: / Sic pretium vitae mors fuit artifici. // Tu quoque dum toto laniatam corpore Romam / Componis miro, Raphael, ingenio: // Atque Urbis lacerum ferro, igni, annisque cadaver / Ad vitam, antiquum iam revocasque decus; // Movisti superûm invidiam; idignataque Mors est, / Te dudum exstinctis reddere posse animam: // Et quod longa dies paullatim aboleverat, hoc te / Mortali spreta lege parare iterum. // Sic miser heu prima cadis intercepte iuventa; / Deberi et Morti nostraque nosque mones» Berni/Castiglione/Della Casa i 995, pp. 53-54.

i 8. Petrarca i996, pp. 270-7i. 
19. Citato da Maier in Castiglione $198 \mathrm{I}^{3}$, p. 599.

20. Per il Cortegiano si veda Honnacker 2002b, pp. 35-78 e la bibliografia ivi riportata.

2i. Castiglione i98 $\mathrm{I}^{3}$, p. 43.

22. Cfr. al riguardo Honnacker 2002b, p. 63 sgg.

23. Castiglione i $98 \mathrm{I}^{3}$, I, Xxix-Xxxix pp. I33-58.

24. Castiglione i98 $\mathrm{I}^{3}$, I, Xxxi-Xxxil, p. I 39.

25. Castiglione i98 ${ }^{3}$, I, xxxix, p. i 56.

26. A tale proposito si veda Honnacker 2002b, p. 58 sgg. Per il Cortegiano come 'dialogo aperto' si confronti anche Roncaccia 2006, p. 52 sgg.

27. Castiglione i968, I, xxxix, pp. 53 sg., r. 3 I-40.

28. Petrarca, Il Canzoniere, CLXXXVII, I-4.

29. Castiglione i98 $\mathrm{I}^{3}$, I, Xlvi, p. 170.

30. Castiglione i $98 \mathrm{I}^{3}$, I, Xlvi, p. $170 \mathrm{sg}$.

31. Bernardo Accolti, I458-i 535 , detto l'Unico Aretino, all'epoca apprezzato per le sue doti di improvvisatore di versi. Compose sessantasei rime per Elisabetta Gonzaga, duchessa di Urbino, sotto il nome di Julia, ma dedicò versi anche ad altre nobildonne, come per esempio ad Isabella d'Aragona e Costanza Vittoria d'Ávalos. Seguendo Cian, Maier cita anche una poesia dell'Unico Aretino a proposito del motivo delle rovine dell'antica Roma nel sonetto Superbi colli, e voi sacre ruine (Castiglione i $98 \mathrm{I}^{3}$, p. 599).

32. Citato da Maier in Castiglione i $98 \mathrm{I}^{3}$, I, IX, p. 98 , nota $\mathrm{I} 3$.

33. Castiglione $198 \mathrm{I}^{3}$, I, x, p. 99. Per le proposte di 'gioco', tra le quali alla fine Emilia Pio sceglie la discussione sulla formazione del 'bon cortigiano', si veda Honnacker 2002b, p. 50 sgg.

34. A proposito del rapporto tra Castiglione e l'Unico Aretino, Cian scrive: «Così aveva cantato, più sgraziatamente, in quei giorni l'Unico Aretino. Che se il Castiglione pensò di rifargli il verso e impartirgli una lezione di gusto, bisogna riconoscere che l'aretino era riuscito a vendicarsi, con una iniezione, sia pure non gravissima, di cattivo gusto» Cian I95 I, pp. 205-206.

35. Cfr. Castiglione i 98 I $^{3}$, I, ix, p. 96 sgg.

36. Al riguardo si vedano i saggi citati nella nota i 2. 